\title{
Time to Treatment Initiation and Survival in Adult Localized High-Grade Bone Sarcoma
}

\author{
Joshua M. Lawrenz $\mathbb{D}^{1},{ }^{1}$ Joseph Featherall, ${ }^{2}$ Gannon L. Curtis, ${ }^{3}$ Jaiben George, ${ }^{4}$ Yuxuan Jin, ${ }^{5}$ \\ Peter M. Anderson, ${ }^{5}$ Dale R. Shepard, ${ }^{5}$ John D. Reith $\left(\mathbb{D},{ }^{5}\right.$ Brian P. Rubin, \\ Lukas M. Nystrom $\mathbb{D}^{5}$ and Nathan W. Mesko ${ }^{5}$
}

${ }^{1}$ Vanderbilt University Medical Center, Nashville, TN 37232, USA

${ }^{2}$ University of Utah Hospital, Salt Lake City, UT 84132, USA

${ }^{3}$ University Health Center, Wayne State University, Detroit, MI 48201, USA

${ }^{4}$ AIIMS Hospital, New Delhi, India

${ }^{5}$ Cleveland Clinic, Cleveland, $\mathrm{OH} 44195$, USA

Correspondence should be addressed to Joshua M. Lawrenz; josh.lawrenz@gmail.com

Received 16 February 2020; Accepted 15 April 2020; Published 5 May 2020

Academic Editor: Valerae O. Lewis

Copyright (c) 2020 Joshua M. Lawrenz et al. This is an open access article distributed under the Creative Commons Attribution License, which permits unrestricted use, distribution, and reproduction in any medium, provided the original work is properly cited.

\begin{abstract}
Objective. Few studies have evaluated the prognostic implication of the length of time from diagnosis to treatment initiation in bone sarcoma. The purpose of this study is to determine if time to treatment initiation (TTI) influences overall survival in adults diagnosed with primary bone sarcoma. Methods. A retrospective analysis of the National Cancer Database identified 2,122 patients who met inclusion criteria with localized, high-grade bone sarcoma diagnosed between 2004 and 2012. TTI was defined as length of time in days from diagnosis to initiation of treatment. Patient, disease-specific, and healthcare-related factors were also assessed for their association with overall survival. Kruskal-Wallis analysis was utilized for univariate analysis, and Cox regression modeling identified covariates associated with overall survival. Results. Any 10-day increase in TTI was not associated with decreased overall survival (hazard ratio $(\mathrm{HR})=1.00 ; P=0.72$ ). No differences in survival were detected at 1 year, 5 years, and 10 years, when comparing patients with TTI $=14,30,60,90$, and 150 days. Decreased survival was significantly associated $(P<0.05)$ with patient ages of $51-70$ years $(\mathrm{HR}=1.66 ; P=0.004)$ and $>71$ years $(\mathrm{HR}=2.89 ; P<0.001)$, Charlson/Deyo score $\geq 2(\mathrm{HR}=2.02$; $P<0.001)$, pelvic tumor site $(\mathrm{HR}=1.58 ; P<0.001)$, tumor size $>8 \mathrm{~cm}(\mathrm{HR}=1.52 ; P<0.001)$, radiation $(\mathrm{HR}=1.81 ; P<0.001)$ as index treatment, and residing a distance of $51-100$ miles from the treatment center $(\mathrm{HR}=1.30 ; P=0.012)$. Increased survival was significantly associated $(P<0.05)$ with chordoma $(\mathrm{HR}=0.27 ; P=0.010)$, chondrosarcoma $(\mathrm{HR}=0.75 ; P=0.002)$, treatment at an academic center $(\mathrm{HR}=0.64 ; P=0.039)$, and a private $(\mathrm{HR}=0.67 ; P=0.006)$ or Medicare $(\mathrm{HR}=0.71 ; P=0.043)$ insurer. A transition in care was not associated with a survival disadvantage $(\mathrm{HR}=0.90 ; P=0.14)$. Conclusions. Longer TTI was not associated with decreased overall survival in localized, high-grade primary bone sarcoma in adults. This is important in counseling patients, who may delay treatment to receive a second opinion or seek referral to a higher volume sarcoma center.
\end{abstract}

\section{Introduction}

Primary bone sarcomas are rare malignancies with a national incidence in the United States of around 3,200 cases annually and a five-year relative survival between 60 and $70 \%$ in localized disease [1]. Prognosis in bone sarcoma is closely correlated with tumor grade and disease stage, which argues for earlier diagnosis and treatment $[2,3]$. Time to treatment initiation (TTI), defined as the duration of time between diagnosis and the initiation of treatment, has become an important quality metric in cancer care, as the length of this time period can potentially affect patient anxiety and outcome. Registry data for breast and head and neck cancers have demonstrated an association between 
increased treatment wait times and decreased survival $[4,5]$. It is arguable that the potential benefits of shorter TTI would apply to most, if not all, cancers, including high-grade bone sarcoma. Despite the obvious benefits of expedited treatment, other factors such as treatment at an established multidisciplinary sarcoma program are believed to positively affect prognosis but may result in a treatment delay due to coordination and transfer of care [6-8]. Thus, the inquiry as to if TTI affects prognosis in bone sarcoma is nuanced, and the rarity of the disease has led to limited data addressing this issue [9].

The National Cancer Database (NCDB) is a high-quality cancer registry that captures data from newly diagnosed cancers in the United States and is of particular value when investigating rare cancers such as bone sarcoma [10]. The NCDB has been utilized to investigate the correlation between time to treatment and survival in other cancer types in effort to reduce delays and improve outcomes $[4,5,11]$. In a recent inquiry of the effect of TTI on survival in soft tissue sarcoma, TTI was found to have minimal effect on overall survival (OS), with a delay of greater than 42 days having a trend toward decreasing survival [7]. No similar studies have been performed with the primary goal to establish this correlation in bone sarcoma.

The primary aim of this study was to determine if TTI influences OS in patients diagnosed with localized, highgrade bone sarcoma. We hypothesized that prolonged TTI would be associated with decreased survival in bone sarcoma patients. Additionally, the secondary aim was to identify patient socioeconomic, tumor-specific, and healthcare-related factors that contribute to bone sarcoma survival.

\section{Methods}

2.1. Database and Selection of Patients. Following approval by our institutional review board, the NCDB was reviewed from 2004 to 2012. Created in 1989 by the American College of Surgeons (ACS) and the Commission on Cancer (CoC), the NCDB captures $70 \%$ of all new United States cancer diagnoses and collects data from over 1,500 cancer centers [12]. The methodology for reporting to the NCDB has been previously described [8]. Adult patients ( $\geq 18$ years old) with bone sarcoma diagnosed between 2004 and 2012 were identified using topographical codes (C40.0-C40.3, C40.8C41.4, C41.8, C41.9) designated by International Classification of Disease for Oncology, Third Edition [ICD-O-3]. A patient also required an ICD-O-3 histology code consistent with a bone sarcoma to be included. These codes identified a total of 13,329 patients with a bone sarcoma. Patients were excluded for the following reasons: (1) lack of follow-up or essential data $(n=1,485)$, (2) American Joint Committee on Cancer (AJCC) Stage IV or unknown stage disease $(n=5,686)$, and (3) well differentiated (grade 1$)$, moderately differentiated (grade 2$)$, or unknown grade $(n=4,036)$. Thus, 2,122 adult patients with localized, high-grade disease were included in the final analysis. Given the significant impact tumor grade and disease stage have been shown to have on survival outcome $[9,13,14]$, this cohort was intentionally limited to patients with high grade, localized disease. The inclusion criteria can be found in Figure 1.

2.2. Outcome Measures. The primary objective of this study was to evaluate the association between TTI and OS in patients with localized, high-grade bone sarcoma. TTI was defined as the time in days between confirmed tissue diagnosis and initiation of any definitive treatment course (surgical resection, systemic chemotherapy, and radiation therapy). Diagnostic or palliative procedures do not qualify as treatment initiation. OS was defined as the time in months from treatment initiation until death or the patient's last follow-up visit. Patient, healthcare, and tumor characteristics (Table 1) were also collected to investigate their associations with patient OS. Patient demographics included age, gender, race, Charlson/Deyo Score (CDS) $(0,1$, or $>2)$, and annual income. It is important to note that annual income is not patient derived data, but rather the mean income reported in the patient's zip code. Tumor factors included histology, primary site, size, grade, clinical stage, and initial definitive treatment modality. Healthcare system factors included treating facility type, insurance provider, distance from the patient's residence to the treating facility, and presence of a transition in care. Patients who received a diagnosis at one facility and had initial treatment commencement at another facility were considered to have a transition in care. Facility type was divided into community cancer programs, comprehensive cancer centers, academic centers, integrated network cancer programs, and other. Community cancer programs are defined as having 100-500 new cancer cases a year, whereas comprehensive cancer programs are defined as diagnosing $>500$ new cancer cases a year. "New cancer cases" are defined as all histologic diagnoses, not exclusively sarcoma. Community programs offer both diagnostic and treatment services, although what specific treatment services offered for rare malignancies such as sarcoma are unknown. Integrated network cancer programs usually have a "unified cancer committee" and consist of "multiple facilities providing comprehensive services" [15]. Academic institutions are defined with the same patient volume definition as a comprehensive cancer center but also have a noted resident/medical education program.

2.3. Statistical Analysis. The number of patients and frequencies for all independent categorical variables were reported. Median TTI was reported given the nonparametric dataset and was compared across different levels of the same categorical variable by using Kruskal-Wallis tests. The relationship between OS and TTI, along with other important secondary covariates such as age, gender, race, and treatment modality were examined with Cox regression modeling. Hazard ratios (HR) and 95\% confidence intervals (CI) were determined for each variable. The TTI variable was entered into the full Cox regression by using four-knot restricted cubic splines to allow for a nonlinear relationship between TTI and the survival outcome [16]. However, the spline effect was not significant. Given the nonsignificant and nonlinear relationship of TTI with survival in all TTI 


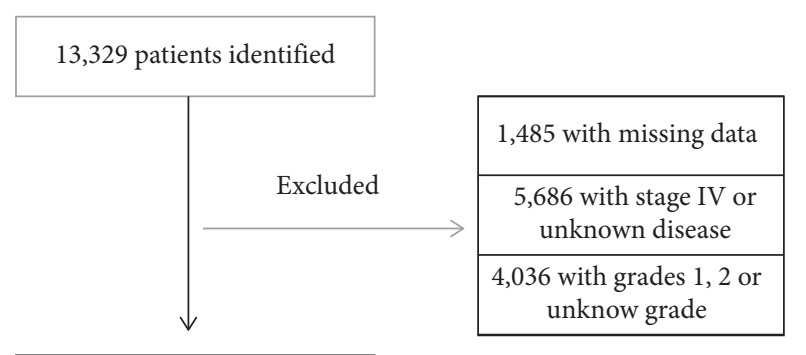

2,122 patients included in analysis

FIGURE 1: Study cohort inclusion criteria.

TABle 1: Demographic data.

\begin{tabular}{|c|c|}
\hline Factor & Total $(N=2,122)$ \\
\hline Time to treatment initiation, days (IQR) & $25.0[12.0,42.0]$ \\
\hline \multicolumn{2}{|l|}{ Age } \\
\hline $18-30$ & $654(30.8 \%)$ \\
\hline $31-50$ & $555(26.2 \%)$ \\
\hline $51-70$ & $627(29.5 \%)$ \\
\hline $71+$ & $286(13.5 \%)$ \\
\hline \multicolumn{2}{|l|}{ Sex } \\
\hline Male & $1,241(58.5 \%)$ \\
\hline Female & $881(41.5 \%)$ \\
\hline \multicolumn{2}{|l|}{ Race } \\
\hline White & $1,735(81.8 \%)$ \\
\hline Black & $258(12.2 \%)$ \\
\hline Other/unknown & $129(6.1 \%)$ \\
\hline \multicolumn{2}{|l|}{ Charlson/Deyo score } \\
\hline 0 & $1,843(86.9 \%)$ \\
\hline 1 & $222(10.5 \%)$ \\
\hline$\geq 2$ & $57(2.7 \%)$ \\
\hline \multicolumn{2}{|l|}{ Histology } \\
\hline Osteosarcoma & $1,217(57.4 \%)$ \\
\hline Chondrosarcoma & $486(22.9 \%)$ \\
\hline Ewing's sarcoma & $195(9.2 \%)$ \\
\hline Chordoma & $17(0.80 \%)$ \\
\hline Other & $207(9.8 \%)$ \\
\hline \multicolumn{2}{|l|}{ Facility type } \\
\hline Comm. cancer prg. & $38(1.8 \%)$ \\
\hline Comprehensive comm. cancer prg. & $286(13.5 \%)$ \\
\hline Academic center & $856(40.3 \%)$ \\
\hline Integrated network cancer prg. & $62(2.9 \%)$ \\
\hline Other/unknown & $880(41.5 \%)$ \\
\hline \multicolumn{2}{|l|}{ Insurance } \\
\hline Uninsured & $120(5.7 \%)$ \\
\hline Private insurance & $1,148(54.1 \%)$ \\
\hline Medicaid & $258(12.2 \%)$ \\
\hline Medicare & $454(21.4 \%)$ \\
\hline Other/unknown & $142(6.7 \%)$ \\
\hline \multicolumn{2}{|l|}{ Income } \\
\hline$<\$ 38,000$ & $404(19.0 \%)$ \\
\hline$\$ 38,000-\$ 47,999$ & $500(23.6 \%)$ \\
\hline$\$ 48,000-\$ 62,999$ & $563(26.5 \%)$ \\
\hline$\$ 63,000+$ & $655(30.9 \%)$ \\
\hline \multicolumn{2}{|l|}{ Distance from facility } \\
\hline$<21$ miles & $974(45.9 \%)$ \\
\hline 21-50 miles & $466(22.0 \%)$ \\
\hline $51-100$ miles & $306(14.4 \%)$ \\
\hline$>100$ miles & $376(17.7 \%)$ \\
\hline
\end{tabular}

TABLE 1: Continued.

\begin{tabular}{lc}
\hline Factor & Total $(N=2,122)$ \\
\hline Transition in care & \\
No & $1,137(53.6 \%)$ \\
Yes & $985(46.4 \%)$ \\
Year of diagnosis & \\
2004 & $160(7.5 \%)$ \\
2005 & $213(10.0 \%)$ \\
2006 & $226(10.7 \%)$ \\
2007 & $202(9.5 \%)$ \\
2008 & $244(11.5 \%)$ \\
2009 & $273(12.9 \%)$ \\
2010 & $274(12.9 \%)$ \\
2011 & $267(12.6 \%)$ \\
2012 & $263(12.4 \%)$ \\
Primary tumor site & \\
Upper extremity & $281(13.2 \%)$ \\
Lower extremity & $985(46.4 \%)$ \\
Pelvis & $344(16.2 \%)$ \\
Other & $512(24.1 \%)$ \\
Tumor size & \\
$\leq 8.0$ cm & $967(45.6 \%)$ \\
$>8.0$ cm & $1,155(54.4 \%)$ \\
Grade & \\
Poorly differentiated & $1,253(59.0 \%)$ \\
Undifferentiated & $869(41.0 \%)$ \\
Clinical staging & \\
Stage I & $239(11.3 \%)$ \\
Stage II & $1,782(84.0 \%)$ \\
Stage III & $101(4.8 \%)$ \\
Sirst-line treatment modality & \\
Radiation & $1,029(48.5 \%)$ \\
Systemic & $920(4.1 \%)$ \\
Multimodal & $13(0.61 \%)$ \\
Vital status & \\
Died & \\
Alive & $(56.6 \%)$ \\
\hline
\end{tabular}

Statistics presented as median [P25, P75] or N (column \%). Community cancer program: between 100 and 500 new cancer cases annually, Comprehensive community cancer program: $>500$ new cancer cases annually, academic center: $>500$ new cancer cases annually and resident/medical education, integrated network: multiple facilities providing comprehensive services with a unified cancer committee. comm., community; prg., program.

cohorts, cubic spline modeling of HR according to TTI as a continuous variable was not performed. After specifying different TTI values (TTI $=14,30,60,90$, and 150 days) and by setting the covariates to their reference levels, the 1-year, 5-year, and 10-year survival probabilities were determined and associated survival curves were plotted. Statistical analyses were completed with SAS software (Version 9.4; Cary, NC). The multivariable cox regression model was built using rms package in $\mathrm{R}$ software (Version 3.4; Vienna, Austria). All tests were two-sided, with an alpha level of 0.05 . $P$ values less than 0.05 were considered significant.

\section{Results}

3.1. TTI and Survival. Overall survival probabilities demonstrated minimal differences at 1 year, 5 years, and 10 years 
at $\mathrm{TTI}=14,30,60,90$, and 150 days (Table 2). Similarly, adjusted survival curves generated by Cox regression modeling were near identical out to 10 years $(\mathrm{HR}=1.00$; $P=0.72$ ) (Figure 2).

3.2. Factors That Influence Survival. Univariate analysis revealed significant differences seen in regard to the relationship of TTI with several secondary variables (Table 3). Multivariable analysis also identified several secondary patient, tumor, treatment, and healthcare system related factors associated with mortality (Table 4). Those that were statistically significant are highlighted in Figure 3. Patient factors such as age between 51 and $70(\mathrm{HR}=1.66 ; P=0.004)$ and age of $71+(\mathrm{HR}=2.89 ; P<0.001)$ and patients with a Charlson/Deyo score $\geq 2(\mathrm{HR}=2.02 ; P<0.001)$ were associated with decreased survival, whereas sex, race, and income were not associated with survival. A diagnosis of chondrosarcoma $(\mathrm{HR}=0.75 ; P=0.002)$, chordoma $(\mathrm{HR}=0.27$; $P=0.01)$, or other bone sarcoma not including Ewing's sarcoma $(\mathrm{HR}=0.75 ; P=0.022)$ all were associated with increased survival when compared to osteosarcoma, whereas tumors located in the pelvis $(\mathrm{HR}=1.58 ; P<0.001)$ and tumors greater than $8 \mathrm{~cm}$ in size $(\mathrm{HR}=1.52 ; P<0.001)$ were associated with decreased survival. Being a distance between 51 and 100 miles from the treatment center $(\mathrm{HR}=1.30$; $P=0.012$ ) compared to being less than 21 miles away was associated with decreased survival, though being greater than 100 miles away had no effect. Any year of diagnosis between 2005 and 2012 compared to 2004 did not influence prognosis. Patients treated at an academic center $(\mathrm{HR}=0.64$; $P=0.039)$ or other noncategorized center $(\mathrm{HR}=0.50$; $P=0.006)$ compared to a community cancer program had an association with increased survival. Patients with private insurance $(\mathrm{HR}=0.65 ; P=0.004)$ or Medicare insurance $(\mathrm{HR}=0.71 ; P=0.043)$ had an association with increased survival. Having a transition in care after diagnosis to another center for treatment did not influence survival outcome $(\mathrm{HR}=0.90 ; P=0.14)$. First-line treatment of radiation therapy $(\mathrm{HR}=1.81 ; P<0.001)$ when compared to surgery as first treatment had an association with decreased survival. Tumor grade and clinical stage did not demonstrate association with survival, as to be expected in a cohort of only high grade, localized bone sarcomas.

\section{Discussion}

These data demonstrate that all cause survival probability at one, five, and ten years after diagnosis was no different when comparing patients with a TTI ranging from 0 to 150 days (five months). Factors found to correlate with survival included patient age, comorbidity index, histologic subtype, primary tumor location and size, initial treatment type, type of insurance, treating facility type, and distance of home residence from the treating facility.

Prior data associating treatment delay with survival outcome in sarcoma is limited, with only a single study that compared a treatment delay of less than or greater than three weeks [9]. The authors concluded no difference on survival
TABLE 2: 1-year, 5-year, and 10-year survival probabilities based upon time to treatment initiation.

\begin{tabular}{lcll}
\hline Time & Survival probability & \multicolumn{2}{c}{$95 \%$ CI } \\
\hline TTI $=14$ & & & \\
1 year & 0.84 & 0.74 & 0.95 \\
5 years & 0.46 & 0.26 & 0.79 \\
10 years & 0.36 & 0.18 & 0.74 \\
TTI = 30 & & & \\
1 year & 0.84 & 0.74 & 0.95 \\
5 years & 0.46 & 0.26 & 0.79 \\
10 years & 0.36 & 0.18 & 0.74 \\
TTI =60 & & & \\
1 year & 0.84 & 0.74 & 0.95 \\
5 years & 0.46 & 0.27 & 0.80 \\
10 years & 0.37 & 0.18 & 0.75 \\
TTI = 90 & & & \\
1 year & 0.84 & 0.75 & 0.95 \\
5 years & 0.46 & 0.27 & 0.80 \\
10 years & 0.37 & 0.18 & 0.75 \\
TTI = 150 & & & \\
1 year & 0.85 & 0.75 & 0.96 \\
5 years & 0.47 & 0.27 & 0.82 \\
10 years & 0.38 & 0.18 & 0.78 \\
\hline
\end{tabular}

TTI: time to treatment initiation; CI: confidence interval.

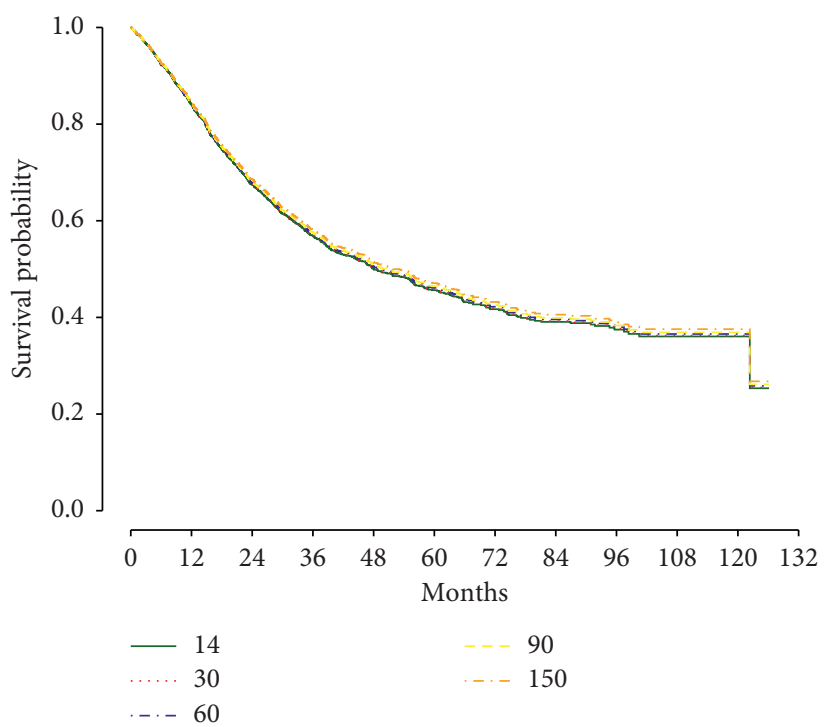

FIgURE 2: Survival curves using different values for time to treatment initiation. This graph demonstrates the near-identical Kaplan-Meier survival curves when comparing patients with a time to treatment initiation of $14,30,60,90$, and 150 days $(\mathrm{HR}=1.00$; $P=0.72$ ).

outcome in their cohort of extremity osteosarcomas. In both breast and head and neck cancers, recent registry data have shown a correlation between increased treatment wait times and decreased survival [4,5]. Nevertheless, given the findings of the present study and previous work in soft tissue sarcoma [7], it remains unclear as to why TTI has little prognostic implication in sarcoma.

Far more studied is the association between time to diagnosis and survival, as delay in diagnosis is the most common reason for litigation related to the treatment of 
TABLE 3: Univariate relationships between factors and time to treatment initiation.

\begin{tabular}{|c|c|c|c|}
\hline Factor & $\mathrm{N}$ & TTI, days median [p25, p75] & $P$ value \\
\hline Age & & & 0.006 \\
\hline $18-30$ & 654 & $21.0[13.0,366.0]$ & \\
\hline $31-50$ & 555 & $28.0[14.0,44.0]$ & \\
\hline $51-70$ & 627 & $26.0[12.0,45.0]$ & \\
\hline $71+$ & 286 & $25.5[7.0,44.0]$ & \\
\hline Sex & & & 0.52 \\
\hline Male & 1241 & $24.0[12.0,42.0]$ & \\
\hline Female & 881 & $26.0[13.0,43.0]$ & \\
\hline Race & & & 0.050 \\
\hline White & 1735 & $25.0[12.0,42.0]$ & \\
\hline Black & 258 & $28.0[22.0,48.0]$ & \\
\hline Other/unknown & 129 & $20.0[7.0,44.0]$ & \\
\hline Charlson/Deyo score & & & 0.18 \\
\hline 0 & 1843 & $25.0[13.0,42.0]$ & \\
\hline 1 & 258 & $22.0[9.0,40.0]$ & \\
\hline$\geq 2$ & 57 & $29.0[7.0,44.0]$ & \\
\hline Histology & & & 0.003 \\
\hline Osteosarcoma & 1217 & $25.0[13.0,40.0]$ & \\
\hline Chondrosarcoma & 486 & $27.0[10.0,48.0]$ & \\
\hline Ewing's sarcoma & 195 & $21.0[11.0,34.0]$ & \\
\hline Chordoma & 17 & $38.0[17.0,77.0]$ & \\
\hline Other & 207 & $29.0[15.0,49.0]$ & \\
\hline Facility type & & & $<0.001$ \\
\hline Comm. cancer prg. & 38 & $32.5[1.00,48.0]$ & \\
\hline Comprehensive comm. cancer Prg. & 286 & $21.5[5.0,40.0]$ & \\
\hline Academic center & 856 & $27.0[14.0,47.0]$ & \\
\hline Integrated network cancer program & 62 & $29.5[11.0,52.0]$ & \\
\hline Other/unknown & 880 & $23.0[13.0,37.0]$ & \\
\hline Insurance & & & $<0.001$ \\
\hline Uninsured & 120 & $27.5[15.0,43.5]$ & \\
\hline Private insurance & 1148 & $23.0[12.0,39.0]$ & \\
\hline Medicaid & 258 & $26.0[13.0,43.0]$ & \\
\hline Medicare & 454 & $27.5[10.0,48.0]$ & \\
\hline Other/unknown & 142 & $35.0[19.0,52.0]$ & \\
\hline Income & & & 0.73 \\
\hline$<\$ 38,000$ & 404 & $25.5[12.0,45.5]$ & \\
\hline$\$ 38,000-\$ 47,999$ & 500 & $24.0[12.0,40.5]$ & \\
\hline$\$ 48,000-\$ 62,999$ & 563 & $24.0[11.0,43.0]$ & \\
\hline$\$ 63,000+$ & 655 & $26.0[14.0,42.0]$ & \\
\hline Distance from facility & & & 0.069 \\
\hline$>21$ miles & 974 & $25.0[12.0,42.0]$ & \\
\hline 21-50 miles & 466 & $24.0[13.0,42.0]$ & \\
\hline $51-100$ miles & 306 & $23.0[9.0,40.0]$ & \\
\hline$>100$ miles & 376 & $28.0[14.5,44.0]$ & \\
\hline Transition in care & & & $<0.001$ \\
\hline No & 1137 & $20.0[8.0,35.0]$ & \\
\hline Yes & 985 & $31.0[19.0,49.0]$ & \\
\hline Year of diagnosis & & & 0.25 \\
\hline 2004 & 160 & $25.5[9.0,42.0]$ & \\
\hline 2005 & 213 & $27.0[14.0,51.0]$ & \\
\hline 2006 & 226 & $24.0[11.0,39.0]$ & \\
\hline 2007 & 202 & $25.5[13.0,43.0]$ & \\
\hline 2008 & 244 & $25.0[13.0,42.0]$ & \\
\hline 2009 & 273 & $23.0[11.0,39.0]$ & \\
\hline 2010 & 274 & $25.5[14.0,41.0]$ & \\
\hline 2011 & 267 & $27.0[14.0,44.0]$ & \\
\hline 2012 & 263 & $25.0[12.0,42.0]$ & \\
\hline Primary tumor site & & & $<0.001$ \\
\hline Upper extremity & 281 & $25.0[14.0,42.0]$ & \\
\hline Lower extremity & 985 & $22.0[12.0,36.0]$ & \\
\hline
\end{tabular}


TABLE 3: Continued.

\begin{tabular}{|c|c|c|c|}
\hline Factor & $\mathrm{N}$ & TTI, days median [p25, p75] & $P$ value \\
\hline Pelvis & 344 & $29.0[15.0,49.0]$ & \\
\hline Other & 512 & $28.0[9.5,48.0]$ & \\
\hline Tumor size & & & 0.48 \\
\hline$\leq 8.0 \mathrm{~cm}$ & 967 & $26.0[12.0,42.0]$ & \\
\hline$>8.0 \mathrm{~cm}$ & 1155 & $25.0[13.0,42.0]$ & \\
\hline Grade & & & 0.63 \\
\hline Poorly differentiated & 1253 & $25.0[11.0,43.0]$ & \\
\hline Undifferentiated & 869 & $26.0[14.0,42.0]$ & \\
\hline Clinical staging & & & 0.10 \\
\hline Stage I & 239 & $28.0[14.0,49.0]$ & \\
\hline Stage II & 1782 & $24.0[12.0,42.0]$ & \\
\hline Stage III & 101 & $27.0[11.0,45.0]$ & \\
\hline First-line treatment modality & & & $<0.001$ \\
\hline Surgery & 1029 & $24.0[6.0,47.0]$ & \\
\hline Radiation & 86 & $34.5[19.0,56.0]$ & \\
\hline Systemic & 994 & $25.0[15.0,37.0]$ & \\
\hline Multimodel & 13 & $39.0[26.0,47.0]$ & \\
\hline
\end{tabular}

$P$ values correspond to the Kruskal-Wallis test. Comm.: community; prg.: program; TTI: time to treatment initiation.

extremity sarcoma [17]. The traditional legal argument is that the increased time allows for a cancer to grow and spread, leading to worse prognosis. Prior studies have evaluated the length of time prior to diagnosis (or duration of symptoms) in bone sarcoma and have demonstrated no significant correlation with survival $[13,14]$. This information is useful when counseling patients who exhibit remorse or anxiety for not presenting to a physician sooner. Considering the lack of correlation between longer duration of symptoms and worsened survival, it is perhaps not surprising that TTI (which is typically a much shorter time period than time to diagnosis, 3 weeks [8] vs. 16 weeks [13]), similarly found no difference. Factors rooted in tumor biology, outside the control of the treating team, are likely a powerful confounding factor in understanding the natural history of primary bone sarcoma.

In a 2019 analysis utilizing the NCDB population, Lawrenz et al. identified patient and disease-specific factors that correlated with TTI in over 13,000 patients with bone sarcoma, highlighting transitions in care from one treating facility to another as being responsible for the greatest increases in TTI [8]. Other factors associated with longer TTI included uninsured or government insurer status, nonwhite race, pelvic tumor location, and treatment at an academic center. A secondary aim of this study was to identify patient, tumor, and healthcare system factors associated with survival. Understanding the overwhelming influence tumor grade and disease stage have been shown to have on prognosis $[9,13,14]$, this cohort was intentionally limited to patients with high grade, localized disease. Similar to prior work, this data reiterates that increased patient age $(>51$ years), increased tumor size $(>8 \mathrm{~cm})$, and pelvic tumor location are correlated with decreased survival, and a diagnosis of chondrosarcoma or chordoma are correlated with increased survival [13]. It was not surprising to learn that patients who underwent radiation therapy as first treatment (86 patients, $4.1 \%$ ) had an associated worse prognosis compared to patients who underwent surgery or systemic therapy first, as we suspect this cohort was likely biased toward unresectable tumors or patients undergoing palliation. Furthermore, patients who lived a distance of 51-100 miles from the treatment center compared to those who lived $<21$ miles away had an increased risk of death, despite having a shorter median TTI (23 days vs. 25 days, respectively). To no surprise, insured status (private insurer or Medicare insurer) when compared to being uninsured was found to be associated with increased survival, similar to recently reported trends seen in prostate, lung, and colorectal cancer [18]. Furthermore, our data supported the previously noted correlation between receiving care at a high-volume facility and improved survival outcome $[7,19,20]$. As well, a transition in care, which previously has been shown to have the greatest correlation with longer TTI [8], was not associated with a survival disadvantage. This supports the concept of referral to a sarcoma referral center with a multidisciplinary treatment team, despite the likely delay in treatment initiation.

This study has several limitations. A retrospective analysis utilizing multivariable regression only allows for determination of correlation between factors and an outcome, not causation. We recognize there are factors not included in our analysis which remain unaccounted for or uncontrolled. To this end, we sought to reduce the potential confounding effect of severity of disease and its known strong correlation with prognosis by restricting this cohort to only patients with localized, high-grade disease. Despite this, we recognize this cohort of bone sarcomas consists of multiple histology types, in which there can be differences amongst individual types on prognosis, which may blunt the effect of treatment delay in the cohort as a whole. Furthermore, national registries are prone to incomplete data reporting and even unknown data collection errors. In this dataset, there were 1,485 patients missing time to treatment data which we excluded. As well, $40 \%$ of patients were categorized as "other/unknown treatment facility type." Given that this was not a critical factor in assessing our 
TABLE 4: Multivariate analysis of factors associated with survival.

\begin{tabular}{|c|c|c|c|c|c|}
\hline \multirow[t]{2}{*}{ Factors } & & \multirow{2}{*}{$\begin{array}{c}\begin{array}{c}\text { Hazard } \\
\text { ratio }\end{array} \\
1.00\end{array}$} & \multicolumn{2}{|c|}{$\begin{array}{c}95 \% \\
\text { hazard } \\
\text { ratio CI }\end{array}$} & \multirow{2}{*}{$\begin{array}{c}P \text { values } \\
0.72\end{array}$} \\
\hline & TTI-a 10-day increase from day 14 & & 0.98 & 1.02 & \\
\hline & TTI-a 10-day increase from day 30 & 1.00 & 0.98 & 1.02 & 0.72 \\
\hline \multirow{4}{*}{ Time to treatments, days } & TTI-a 10-day increase from day 60 & 1.00 & 0.98 & 1.02 & 0.72 \\
\hline & TTI-a 10-day increase from day 90 & 1.00 & 0.98 & 1.02 & 0.72 \\
\hline & TTI-a 10-day increase from day 150 & 1.00 & 0.98 & 1.02 & 0.72 \\
\hline & $31-50$ vs. $18-30$ & 1.16 & 0.88 & 1.51 & 0.29 \\
\hline \multirow[t]{2}{*}{ Age group, years } & $51-70$ vs. $18-30$ & 1.66 & 1.17 & 2.34 & 0.004 \\
\hline & $71+$ vs. $18-30$ & 2.89 & 1.95 & 4.28 & $<0.001$ \\
\hline Sex & Female vs. male & 0.94 & 0.82 & 1.07 & 0.33 \\
\hline \multirow{2}{*}{ Race } & Black vs. white & 0.94 & 0.75 & 1.17 & 0.59 \\
\hline & Other/unknown vs. white & 0.98 & 0.73 & 1.31 & 0.88 \\
\hline \multirow{2}{*}{ Charlson/Deyo score } & 1 vs. 0 & 1.11 & 0.90 & 1.36 & 0.34 \\
\hline & $\geq 2$ vs. 0 & 2.02 & 1.45 & 2.81 & $<0.001$ \\
\hline \multirow{4}{*}{ Income } & $\$ 38,000-\$ 47,999$ vs. $<\$ 38,000$ & 1.08 & 0.88 & 1.33 & 0.45 \\
\hline & $\$ 48,000-\$ 62,999$ vs. $<\$ 38,000$ & 1.03 & 0.84 & 1.27 & 0.75 \\
\hline & $\$ 63,000+$ vs. $<\$ 38,000$ & 1.04 & 0.84 & 1.29 & 0.70 \\
\hline & Private insurance vs. uninsured & 0.65 & 0.49 & 0.87 & 0.004 \\
\hline \multirow{3}{*}{ Insurance } & Medicaid vs. uninsured & 0.85 & 0.61 & 1.18 & 0.33 \\
\hline & Medicare vs. uninsured & 0.71 & 0.51 & 0.99 & 0.043 \\
\hline & Other/unknown vs. uninsured & 0.75 & 0.51 & 1.10 & 0.15 \\
\hline \multirow{5}{*}{ Facility type } & $\begin{array}{c}\text { Comprehensive community cancer program vs. community cancer } \\
\text { program }\end{array}$ & 0.89 & 0.58 & 1.39 & 0.62 \\
\hline & Academic center vs. community cancer program & 0.64 & 0.41 & 0.98 & 0.039 \\
\hline & Integrated network cancer program vs. community cancer program & 0.99 & 0.58 & 1.68 & 0.97 \\
\hline & Other/unknown vs. community cancer program & 0.50 & 0.30 & 0.82 & 0.006 \\
\hline & $21-50$ miles vs. $<21$ miles & 1.17 & 0.98 & 1.39 & 0.088 \\
\hline \multirow[t]{2}{*}{ Distance from facility } & $51-100$ miles vs. $<21$ miles & 1.30 & 1.06 & 1.59 & 0.012 \\
\hline & $>100$ miles vs. $<21$ miles & 1.08 & 0.88 & 1.33 & 0.44 \\
\hline \multirow[t]{4}{*}{ Transition in care } & Yes vs. No & 0.90 & 0.78 & 1.04 & 0.14 \\
\hline & 2005 vs. 2004 & 1.06 & 0.79 & 1.41 & 0.71 \\
\hline & 2006 vs. 2004 & 0.84 & 0.63 & 1.13 & 0.26 \\
\hline & 2007 vs. 2004 & 1.18 & 0.88 & 1.58 & 0.27 \\
\hline \multirow{5}{*}{ Year of diagnosis } & 2008 vs. 2004 & 0.84 & 0.62 & 1.13 & 0.25 \\
\hline & 2009 vs. 2004 & 0.90 & 0.67 & 1.21 & 0.48 \\
\hline & 2010 vs. 2004 & 1.00 & 0.74 & 1.35 & 0.99 \\
\hline & 2011 vs. 2004 & 1.22 & 0.90 & 1.65 & 0.19 \\
\hline & 2012 vs. 2004 & 1.06 & 0.76 & 1.47 & 0.73 \\
\hline \multirow{3}{*}{$\begin{array}{l}\text { First-line treatment } \\
\text { modality }\end{array}$} & Radiation vs. surgery & 1.81 & 1.35 & 2.42 & $<0.001$ \\
\hline & Systemic vs. surgery & 1.17 & 0.99 & 1.39 & 0.06 \\
\hline & Multimodal vs. surgery & 0.60 & 0.24 & 1.49 & 0.27 \\
\hline \multirow{4}{*}{ Histology } & Chondrosarcoma vs. osteosarcoma & 0.75 & 0.62 & 0.90 & 0.002 \\
\hline & Ewing's sarcoma vs. osteosarcoma & 0.82 & 0.62 & 1.09 & 0.17 \\
\hline & Chordoma vs. osteosarcoma & 0.27 & 0.10 & 0.73 & 0.01 \\
\hline & Other vs. osteosarcoma & 0.75 & 0.59 & 0.96 & 0.022 \\
\hline \multirow{3}{*}{ Primary tumor site } & Lower extremity vs. upper extremity & 0.92 & 0.75 & 1.13 & 0.42 \\
\hline & Pelvis vs. upper extremity & 1.58 & 1.26 & 1.99 & $<0.001$ \\
\hline & Other vs. upper extremity & 1.05 & 0.83 & 1.32 & 0.70 \\
\hline Tumor size & $>8.0 \mathrm{~cm}$ vs. $\leq 8.0 \mathrm{~cm}$ & 1.52 & 1.32 & 1.76 & $<0.001$ \\
\hline Grade & Undifferentiated vs. poorly differentiated & 1.07 & 0.94 & 1.23 & 0.31 \\
\hline \multirow{2}{*}{ Clinical staging } & Stage II vs. stage I & 0.96 & 0.77 & 1.20 & 0.72 \\
\hline & Stage III vs. stage I & 0.97 & 0.69 & 1.38 & 0.87 \\
\hline
\end{tabular}

TTI: time to treatment initiation; CI: confidence interval.

primary endpoint, we included these patients for the sake of increased sample size, though making conclusions regarding this specific variable more difficult to interpret. Despite this, when studying a rare disease such as sarcoma, tools such as the NCDB though imperfect provide a large cohort to investigate important questions for the purposes of data 


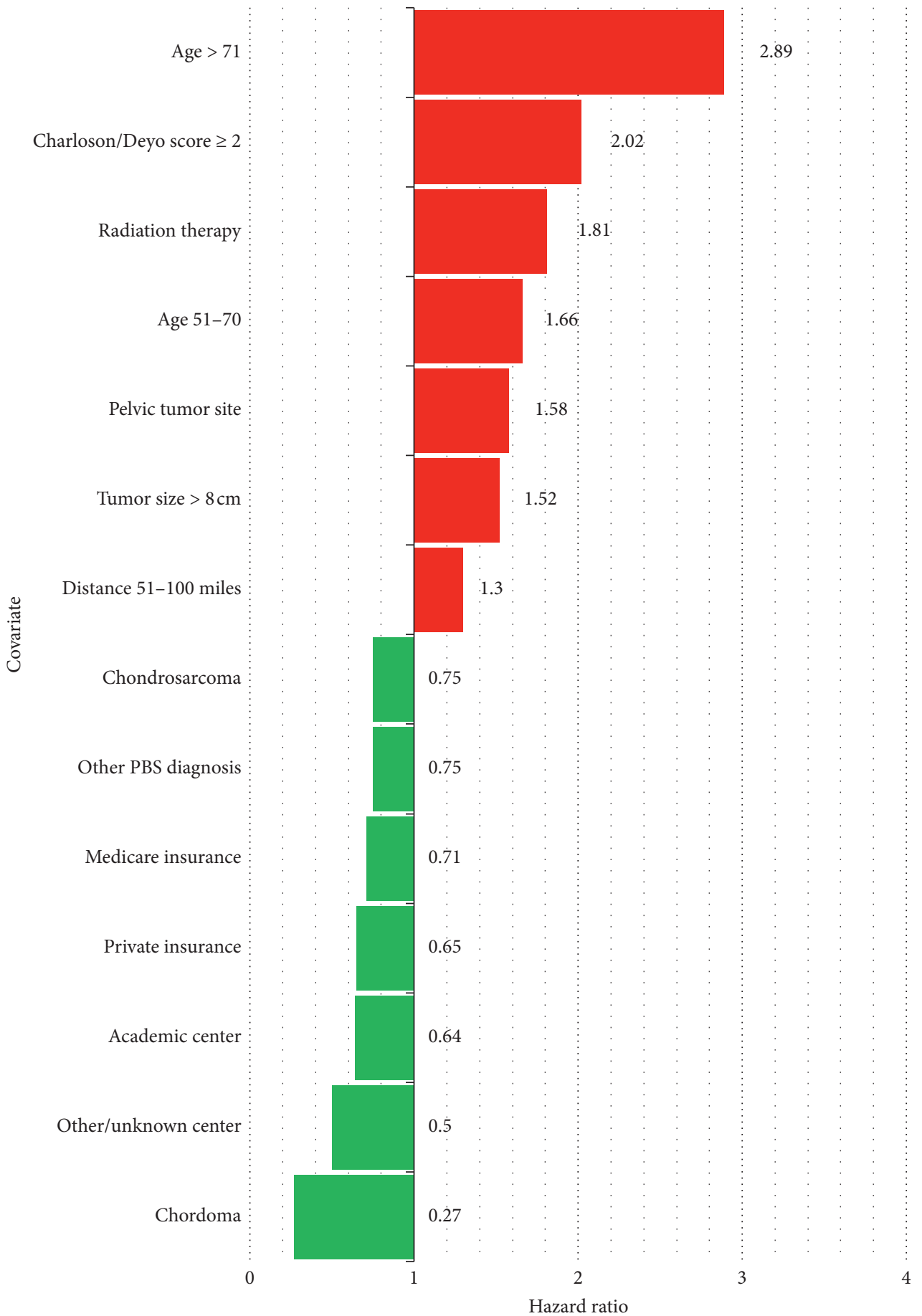

Figure 3: Comparison of relative association between covariates and survival. Only covariates with statistically significant higher (red) or lower (green) HR are shown.

description and hypothesis generation. These limitations could be largely improved upon with a multi-institutional prospective registry effort focused on sarcoma diagnoses.

In conclusion, this analysis of the NCDB from 2004 to 2012 demonstrates TTI does not correlate with overall survival in localized, high-grade primary bone sarcoma in adults. The primary and secondary conclusions of this data suggest that factors inherent to the patient, disease process, and treating facility are likely more integral to overall prognosis, rather than the length of time from when a diagnosis is made and when treatment is initiated. This is important in counseling patients, who may delay treatment to receive a second opinion or seek referral to a higher volume sarcoma center.

\section{Data Availability}

The data used to support the findings of this study are included within the article. 


\section{Conflicts of Interest}

The authors declare that there are no conflicts of interest related to this work.

\section{References}

[1] N. Howlader, A. Noone, and M. Krapcho, SEER Cancer Statistics Review, National Cancer Institute, Bethesda, MD, USA, 2017.

[2] R. J. Grimer, "Size matters for sarcomas!" The Annals of The Royal College of Surgeons of England, vol. 88, no. 6, pp. 519-524, 2006.

[3] R. J. Grimer and T. W. R. Briggs, "Earlier diagnosis of bone and soft-tissue tumours," The Journal of Bone and Joint Surgery. British Volume, vol. 92, no. 11, pp. 1489-1492, 2010.

[4] R. J. Bleicher, K. Ruth, E. R. Sigurdson et al., "Time to surgery and breast cancer survival in the United States," JAMA Oncology, vol. 2, no. 3, pp. 330-339, 2016.

[5] C. T. Murphy, T. J. Galloway, E. A. Handorf et al., "Survival impact of increasing time to treatment initiation for patients with head and neck cancer in the United States," Journal of Clinical Oncology, vol. 34, no. 2, pp. 169-178, 2016.

[6] O. Zaikova, K. Sundby Hall, E. Styring et al., "Referral patterns, treatment and outcome of high-grade malignant bone sarcoma in Scandinavia-SSG Central Register 25 years' experience," Journal of Surgical Oncology, vol. 112, no. 8, pp. 853-860, 2015.

[7] J. Featherall, G. L. Curtis, J. M. Lawrenz et al., "Time to treatment initiation and survival in adult localized, high-grade soft tissue sarcoma," Journal of Surgical Oncology, vol. 120, no. 7, pp. 1241-1251, 2019.

[8] J. M. Lawrenz, G. L. Curtis, J. F. Styron et al., "Adult primary bone sarcoma and time to treatment initiation: an analysis of the National Cancer Database," Sarcoma, vol. 2018, Article ID 1728302, 9 pages, 2018.

[9] S. S. Bielack, B. Kempf-Bielack, G. Delling et al., "Prognostic factors in high-grade osteosarcoma of the extremities or trunk: an analysis of 1,702 patients treated on neoadjuvant cooperative osteosarcoma study group protocols," Journal of Clinical Oncology, vol. 20, no. 3, pp. 776-790, 2002.

[10] T. A. Damron, W. G. Ward, and A. Stewart, "Osteosarcoma, chondrosarcoma, and Ewing's sarcoma," Clinical Orthopaedics and Related Research, vol. 459, pp. 40-47, 2007.

[11] C. T. Murphy, T. J. Galloway, E. A. Handorf et al., "Increasing time to treatment initiation for head and neck cancer: an analysis of the National Cancer Database," Cancer, vol. 121, no. 8, pp. 1204-1213, 2015.

[12] S. Mohanty and K. Y. Bilimoria, "Comparing national cancer registries: the National Cancer Data Base (NCDB) and the surveillance, epidemiology, and end results (SEER) program," Journal of Surgical Oncology, vol. 109, no. 7, pp. 629-630, 2014.

[13] J. M. Lawrenz, J. F. Styron, M. Parry, R. J. Grimer, and N. W. Mesko, "Longer duration of symptoms at the time of presentation is not associated with worse survival in primary bone sarcoma," The Bone \& Joint Journal, vol. 100, no. 5, pp. 652-661, 2018.

[14] B. T. Rougraff, K. Davis, and J. Lawrence, "Does length of symptoms before diagnosis of sarcoma affect patient survival?" Clinical Orthopaedics and Related Research, vol. 462, pp. 181-189, 2007.

[15] National Cancer Data Base-Data Dictionary PUF, 2014.
[16] A. Jemal, R. C. Tiwari, T. Murray et al., "Cancer statistics, 2004," CA: A Cancer Journal for Clinicians, vol. 54, no. 1, pp. 8-29, 2004.

[17] N. W. Mesko, J. L. Mesko, L. M. Gaffney, J. L. Halpern, H. S. Schwartz, and G. E. Holt, "Medical malpractice and sarcoma care-a thirty-three year review of case resolutions, inciting factors, and at risk physician specialties surrounding a rare diagnosis," Journal of Surgical Oncology, vol. 110, no. 8, pp. 919-929, 2014.

[18] L. Ellis, A. J. Canchola, D. Spiegel, U. Ladabaum, R. Haile, and S. L. Gomez, "Trends in cancer survival by health insurance status in California from 1997 to 2014," JAMA Oncology, vol. 4, no. 3, pp. 317-323, 2018.

[19] J. C. Gutierrez, E. A. Perez, F. L. Moffat, A. S. Livingstone, D. Franceschi, and L. G. Koniaris, "Should soft tissue sarcomas be treated at high-volume centers?" Annals of Surgery, vol. 245, no. 6, pp. 952-958, 2007.

[20] A. L. Lazarides, D. L. Kerr, D. P. Nussbaum et al., "Soft tissue sarcoma of the extremities," Clinical Orthopaedics and Related Research, vol. 477, no. 4, pp. 718-727, 2019. 